\title{
Linking confinement to spectral properties of the Dirac operator
}

\author{
Christof Gattringer \\ Institut für Physik, FB Theoretische Physik, Universität Graz, A-8010 Graz, Austria
}

\begin{abstract}
We represent Polyakov loops and their correlators as spectral sums of eigenvalues and eigenmodes of the lattice Dirac operator. The deconfinement transition of pure gauge theory is characterized as a change in the response of moments of eigenvalues to varying the boundary conditions of the Dirac operator. We argue that the potential between static quarks is linked to spatial correlations of Dirac eigenvectors.
\end{abstract}

PACS numbers: 12.38.Aw, 11.15.Ha, 11.10.Wx

\section{Introductory remarks}

Confinement and chiral symmetry breaking are two of the central features of QCD. At the QCD finite temperature transition chiral symmetry is restored and the theory deconfines. Numerical simulations in lattice QCD indicate that the critical temperature $T_{c}$ is the same for both transitions. Thus it is widely believed that there must be a mechanism linking the two phenomena. However, so far there is no generally accepted picture for such a link.

For chiral symmetry breaking an important connection between the order parameter, the chiral condensate, and spectral properties of the Dirac operator is known. The Banks-Casher formula [1] links the chiral condensate to the density of Dirac eigenvalues at the origin. Building on this connection it has been suggested [2] that a liquid of objects with topological charge, e.g., instantons, could lead to a non-vanishing density of eigenvalues and thus such topological objects are candidates for vacuum excitations responsible for chiral symmetry breaking. Above $T_{c}$ the topological objects are expected to rearrange and a spectral gap opens up. The chiral condensate vanishes and chiral symmetry is restored.

Concerning confinement so far no signature in spectral properties of the Dirac operator is known. On the other hand it is obvious that such signatures must exist. The inverse Dirac operator, i.e., the quark propagator, clearly knows about confinement properties. In this letter we present an attempt to identify spectral signatures of the Dirac operator which are related to confinement.

Our starting point are Polyakov loops on a euclidean lattice. A Polyakov loop is defined as the ordered product of temporal link variables at a fixed spatial position $\vec{x}$,

$$
L(\vec{x})=\operatorname{tr}_{c} \prod_{s=1}^{N} U_{4}(\vec{x}, s),
$$

where the $N$ denotes the number of lattice points in time direction and $\operatorname{tr}_{c}$ is the trace over color indices. For pure gauge theory the action is invariant under center rotations, while the Polyakov loop is not. The QCD phase transition can be viewed as spontaneous breaking of the center symmetry and the Polyakov loop is the corresponding order parameter [3]. In the confined phase it vanishes, but above $T_{c}$, where the theory deconfines, the Polyakov loop acquires a non-vanishing expectation value. When fermions are coupled, the center symmetry is broken explicitly by the Dirac operator and the Polyakov loop cannot be used as order parameter then. In the dynamical case correlators of Polyakov loops are related to the potential between static quark sources and thus linked to confinement.

Working in the lattice regularization, we express the Polyakov loop and its correlators as a spectral sum of eigenvalues and eigenvectors of the Dirac operator with different boundary conditions. If the Polyakov loop is averaged over space, our formula contains only moments of eigenvalues. The deconfining transition of QCD can then be seen as a change in the response of these moments to changing boundary conditions of the Dirac operator. We furthermore show, that the static potential is connected to spatial correlations of the eigenvectors.

\section{Dirac operator and Polyakov loops}

To be specific, we work on a lattice of size $L^{3} \times N$ and for the gauge field ( $\mathrm{SU}(n), n$ arbitrary) use boundary conditions which are periodic in all four directions. We base our discussion on the Wilson Dirac operator

$$
D(x \mid y)=(4+m) \delta_{x, y}-\frac{1}{2} \sum_{\mu= \pm 1}^{ \pm 4}\left[1 \mp \gamma_{\mu}\right] U_{\mu}(x) \delta_{x+\hat{\mu}, y}
$$

where we use $U_{-\mu}(x) \equiv U_{\mu}(x-\hat{\mu})^{\dagger}$. At the moment we consider boundary conditions for the Dirac operator which are periodic for all four directions. We remark that our construction goes through unchanged when a chemical potential is coupled in the standard way [4].

The hopping terms of the Wilson Dirac operator (2) connect nearest neighbors. When powers of $D$ are considered, these terms combine to chains of hops on the lattice. Along these chains products of the link variables $U_{\mu}(x)$ and of the matrices $\left[1 \mp \gamma_{\mu}\right] / 2$ are collected. Taking the $m$-th power will give rise to chains with a maximal length of $m$ steps (in chains shorter than $m$ the on-site term appears as a factor). We now consider the $N$-th power of $D$, where $N$ is the temporal extent of our lattice. Thus we will encounter chains with a maximum length of $N$. Furthermore we set the two space-time arguments of $D$ to the same value, $y=x$, such that we pick up only closed chains, i.e., loops starting and ending at 
$x$. Among these are the loops where only hops in time direction occur such that they close around compact time. We obtain (the ${ }^{*}$ denotes complex conjugation)

$$
\begin{aligned}
& \operatorname{tr}_{d} \operatorname{tr}_{c}\left[D^{N}(\vec{x}, t \mid \vec{x}, t)\right]=\operatorname{tr}_{d}\left[\frac{1-\gamma_{4}}{2}\right]^{N} \operatorname{tr}_{c} \prod_{s=1}^{N} U_{4}(\vec{x}, s) \\
& +\operatorname{tr}_{d}\left[\frac{1+\gamma_{4}}{2}\right]^{N} \operatorname{tr}_{c} \prod_{s=0}^{N-1} U_{4}(\vec{x}, N-s)^{\dagger}+\text { other loops } \\
& =2 L(\vec{x})+2 L(\vec{x})^{*}+\text { other loops } .
\end{aligned}
$$

Here $\operatorname{tr}_{d}$ and $\operatorname{tr}_{c}$ denote the traces over Dirac and color indices. Evaluating the Dirac trace is straightforward, due to the projector property $\left(\left[1 \mp \gamma_{4}\right] / 2\right)^{2}=\left[1 \mp \gamma_{4}\right] / 2$. We stress that the forward and backward running Polyakov loops are the only ones that wind non-trivially around the compact time direction.

We now explore the fact that the Polyakov loops respond differently to a change of the boundary conditions compared to other, non-winding loops. We can change the temporal boundary condition of the Dirac operator by multiplying all temporal link variables at $t=N$ with some phase factor $z \in \mathbb{C},|z|=1$,

$$
U_{4}(\vec{x}, N) \rightarrow z U_{4}(\vec{x}, N) \quad \forall \vec{x} .
$$

We evaluate the left-hand side of (3) a second time, now using the Dirac operator in the field transformed according to (4), which we denote as $D_{z}$. We obtain:

$\operatorname{tr}_{d, c}\left[D_{z}^{N}(\vec{x}, t \mid \vec{x}, t)\right]=z 2 L(\vec{x})+z^{*} 2 L(\vec{x})^{*}+$ other loops.

Only the two Polyakov loops which wind non-trivially are altered when changing the boundary condition. All other loops cross the last temporal link, where we put our boundary condition, equally often in both directions (or not at all). Since forward and backward oriented link variables are conjugate to each other the phase factors cancel. This fact is used to get rid of the trivial loops:

$$
\begin{gathered}
\frac{1}{4}\left(\operatorname{tr}_{d, c}\left[D^{N}(\vec{x}, t \mid \vec{x}, t)\right]-\operatorname{tr}_{d, c}\left[D_{z}^{N}(\vec{x}, t \mid \vec{x}, t)\right]\right) \\
=\frac{1-z}{2} L(\vec{x})+\frac{1-z^{*}}{2} L(\vec{x})^{*} .
\end{gathered}
$$

Note that the right-hand side is independent of $t$ and thus the left-hand side can be evaluated at arbitrary $t$. We use this freedom and average over $t$, a step which will be convenient later. It is easy to see that combining periodic boundary conditions with $z= \pm$ i boundary conditions (subscripts \pm ) gives the Polyakov loop:

$$
\begin{gathered}
L(\vec{x})=\frac{1}{8 N} \sum_{t=1}^{N}\left(2 \operatorname{tr}_{d, c}\left[D^{N}(\vec{x}, t \mid \vec{x}, t)\right]-\right. \\
\left.(1+\mathrm{i}) \operatorname{tr}_{d, c}\left[D_{+}^{N}(\vec{x}, t \mid \vec{x}, t)\right]-(1-\mathrm{i}) \operatorname{tr}_{d, c}\left[D_{-}^{N}(\vec{x}, t \mid \vec{x}, t)\right]\right) .
\end{gathered}
$$

We remark that the choice $z= \pm \mathrm{i}$ is not particularly special and also combinations of other phases can project out the Polyakov loop. If one is only interested in the real part of the Polyakov loop, already the combination of periodic and anti-periodic $(z=-1)$ temporal boundary conditions is sufficient as can be seen from Eq. (6).

\section{Spectral representation of Polyakov loops}

We now express the Dirac operator using the spectral theorem. Since the Wilson Dirac operator is not a normal operator it cannot be unitarily diagonalized and we have to use left- and right-eigenvectors. We denote them by $\vec{v}_{L}$ and $\vec{v}_{R}$, with $\vec{v}_{L}$ being a row vector, while $\vec{v}_{R}$ is a column vector. They obey $\vec{v}_{L, \lambda} D=\lambda \vec{v}_{L, \lambda}$ and $D \vec{v}_{R, \lambda}=\lambda \vec{v}_{R, \lambda}$. The spectrum is the same for both eigenvalue problems, however, not the eigenvectors. The eigenvectors form a bi-orthonormal set, $\vec{v}_{L, i} \cdot \vec{v}_{R, j}=\delta_{i j}$, and the spectral representation of the Dirac operator and its $N$-th power respectively, are given by

$$
D=\sum_{\lambda} \lambda \vec{v}_{R, \lambda} \otimes \vec{v}_{L, \lambda}, D^{N}=\sum_{\lambda} \lambda^{N} \vec{v}_{R, \lambda} \otimes \vec{v}_{L, \lambda} .
$$

We remark that for a Dirac operator which is $\gamma_{5^{-}}$ hermitian (such as the Wilson operator we use here) the situation is simplified further: A $\gamma_{5}$-hermitian operator obeys $\gamma_{5} D=D^{\dagger} \gamma_{5}$, implying that the left-eigenvector $\vec{v}_{L, \lambda}$ with eigenvalue $\lambda$ is related to the right-eigenvector with eigenvalue $\lambda^{*}$ (for a $\gamma_{5}$-hermitian Dirac operator the eigenvalues come in complex conjugate pairs) via $\vec{v}_{L, \lambda}=\vec{v}_{R, \lambda^{*}}^{\dagger} \gamma_{5}$, and, after suitable normalization, one can work with the right-eigenvectors alone.

Inserting the spectral sum (8) into (7) we end up with our final expression for the local Polyakov loop:

$$
\begin{gathered}
L(\vec{x})=\frac{1}{8 N}\left(2 \sum_{\lambda} \lambda^{N} \rho(\vec{x})_{\lambda}\right. \\
\left.(1+\mathrm{i}) \sum_{\lambda_{+}} \lambda_{+}^{N} \rho_{+}(\vec{x})_{\lambda_{+}}-(1-\mathrm{i}) \sum_{\lambda_{-}} \lambda_{-}^{N} \rho_{-}(\vec{x})_{\lambda_{-}}\right) .
\end{gathered}
$$

The first sum is over the eigenvalues $\lambda$ obtained with periodic boundary conditions, while the second and third sums are for the cases with phase factors $\pm \mathrm{i}$. We have defined the densities $\rho$ and $\rho_{ \pm}$for these boundary conditions (color and Dirac indices are summed) as:

$$
\begin{aligned}
\rho(\vec{x})_{\lambda} & =\sum_{t=1}^{N} \vec{v}_{L, \lambda}(\vec{x}, t) \cdot \vec{v}_{R, \lambda}(\vec{x}, t), \\
\rho_{ \pm}(\vec{x})_{\lambda_{ \pm}} & =\sum_{t=1}^{N} \vec{v}_{L, \lambda_{ \pm}}(\vec{x}, t) \cdot \vec{v}_{R, \lambda_{ \pm}}(\vec{x}, t) .
\end{aligned}
$$

We remark that both, the eigenvalues as well as the densities $\rho, \rho_{z}$ are gauge invariant. Thus gauge invariance is explicitly manifest in (9), (10) as it should be, since the formula contains no approximations whatsoever.

When using the Polyakov loop as an order parameter for confinement (pure gauge theory), it is usually averaged over the spatial sites $\vec{x}$ to improve statistics,

$$
P \equiv \frac{1}{V_{3}} \sum_{\vec{x}} L(\vec{x})
$$


where $V_{3}$ denotes the spatial volume. When summing over spatial indices as well, the densities (10) turn into the matrix elements of left- and right-eigenvectors with $i=j$ and thus the summed densities equal 1. We obtain for the averaged Polyakov loop,

$$
P=\frac{1}{8 V}\left(2 \sum_{\lambda} \lambda^{N}-(1+\mathrm{i}) \sum_{\lambda_{+}} \lambda_{+}^{N}-(1-\mathrm{i}) \sum_{\lambda_{-}} \lambda_{-}^{N}\right),
$$

where $V=L^{3} N$ is the total number of lattice points. The equation expresses the Polyakov loop through moments of the Dirac eigenvalues at different boundary conditions. We remark that the individual sums are real since the eigenvalues come in complex conjugate pairs.

Let us add a few remarks on the case when an exactly chiral lattice Dirac operator is used. A chiral operator obeys the Ginsparg Wilson [5] equation, $\gamma_{5} D+D \gamma_{5}=$ $D \gamma_{5} D$, which governs chiral symmetry on the lattice. All the properties of the Dirac operator we have used so far still hold. There is, however, an important qualitative difference when using a Ginsparg Wilson Dirac operator. A solution of the Ginsparg Wilson equation is not ultra-local, i.e., $D(x \mid y)$ contains not only nearest neighbor hops, but paths of all lengths. The coefficients for the paths decrease exponentially with their length. The existence of paths of all lengths implies that $D^{N}$ will not only give rise to non-trivially winding loops that are straight lines in time direction, but also "dressed" Polyakov loops taking some detour in spatial directions. These dressed loops are exponentially suppressed with length (as long as one keeps away from the Aoki phase - see [ $\underline{6}]$ ). However, also the dressed Polyakov loops are not invariant under $Z_{N}$ transformations and thus are equally well suited order parameters as are the straight Polyakov loops.

\section{Discussion of the spectral sums}

We begin the interpretation of our formulae with the spectral sum (12) for the averaged Polyakov loop $P$. The right-hand side expresses the Polyakov loop as linear combination of the $N$-th moments of the eigenvalues of the Dirac operator at different boundary conditions. When crossing the QCD phase transition the Polyakov loop acquires a non-vanishing expectation value (pure gauge theory), and the interplay of the eigenvalues at different boundary conditions must change at $T_{c}$.

An interesting question is which part of the Dirac spectrum carries most of the signal for the Polyakov loop. The infrared part of the spectrum (small eigenvalues) is known to undergo a pronounced change as one crosses from the confining to the deconfined phase: A gap opens up in the spectrum, the density of eigenvalues near the origin vanishes and, according to the Banks-Casher formula, chiral symmetry is restored. The size of the gap depends on the relative phase of the Polyakov loop and the Dirac boundary condition [7] .

Although the low-lying eigenvalues undergo a dramatic change, it is not clear whether they give a sizable contri- bution to the Polyakov loop: Due to the large power $N$, the small eigenvalues are strongly suppressed relative to the bulk of the spectrum where eigenvalues of $\mathcal{O}(1)$ occur (in lattice units). On the other hand, since the hopping term changes sign under a staggered sign transformation, for each eigenvalue $\lambda$ also $8-\lambda$ is an eigenvalue. These mirror images undergo the same drastic change at the phase transition, but are not suppressed. Thus small eigenvalues could contribute indirectly through their mirror images at the cutoff. A second important aspect is that the density of eigenvalues increases roughly as $|\lambda|^{3}$. Thus the infrared part of the spectrum and its mirror image at $8-\lambda$ have only a small weight. How much each part of the spectrum contributes to the spectral sum (12) can probably only be decided numerically.

In a first test we summed up the lowest 100 eigenvalues of the chirally improved lattice Dirac operator 8 for quenched $\mathrm{SU}(3)$ configurations on $20^{3} \times 6$ lattices. This was done for temperatures below and above $T_{c}$ and in both cases summing up the low-lying modes gave only a small fraction of the true Polyakov loop $P$ as determined directly from the link variables. It is remarkable, however, that in all cases we checked for $T>T_{c}$, the spectral sum (12) evaluated with even only the 50 lowest eigenvalues already gets the phase of the Polyakov loop right (the $Z_{3}$ symmetry gives rise to 3 preferred phases of the Polyakov loop expectation value). Unfortunately very little is known about the ultraviolet part of the spectrum. Numerical evaluation of the eigenvalue spectrum is usually restricted to the infrared part or to very small lattices due to the immense computational cost of a complete diagonalization. Random matrix theory, which is very successful in describing the infrared part of the spectrum, is valid only up to the Thouless energy and provides no insight to the ultraviolet part of the spectrum.

We now turn to interpreting Eq. (9) which is the spectral sum for the local Polyakov loop at $\vec{x}$. In its local form the Polyakov loop $L(\vec{x})$ can be used for computing the potential $V(r)$ between static sources $(r \equiv|\vec{x}-\vec{y}|)$,

$$
\left\langle L(\vec{x}) L(\vec{y})^{*}\right\rangle \propto \exp (-N V(r)) \sim \exp (-\sigma N r),
$$

where the last expression is the behavior for large $r$, with $\sigma$ denoting the string tension. When inserting the spectral sum (9) in the correlator (13), one sees that the static potential is related to correlations of the densities $\rho$ and $\rho_{ \pm}$. In particular correlations of the form, $\left\langle\rho(\vec{x})_{\lambda} \rho(\vec{y})_{\lambda^{\prime}}^{*}\right\rangle,\left\langle\rho(\vec{x})_{\lambda} \rho_{ \pm}(\vec{y})_{\lambda_{ \pm}^{\prime}}^{*}\right\rangle,\left\langle\rho_{ \pm}(\vec{x})_{\lambda_{ \pm}} \rho_{ \pm}(\vec{y})_{\lambda_{ \pm}^{\prime}}^{*}\right\rangle$, build up $\exp (-N V(|\vec{x}-\vec{y}|))$. In the confining phase the string tension $\sigma$ is non-vanishing, such that the density correlations are expected to decay exponentially with $|\vec{x}-\vec{y}|$ (until string breaking sets in). In the deconfined phase one has $\sigma=0$ and the decay of the eigenvector density correlators should show a power law behavior.

Finally, let us address another possible application of our result: The spectral representation (9) of $L(\vec{x})$ might be an interesting observable also for individual gauge configurations. It provides a filter for analyzing topological 
infrared structures of the gauge field. Expressing gluonic observables in terms of spectral sums and truncating these sums has been applied before (for examples see, e.g., $[9]$ ). The technique is sometimes referred to as "low eigenmode filtering". It makes use of the fact that the low-lying modes of the Dirac operator are an efficient filter for infrared properties of the gauge field. In a thermalized configuration, i.e., one not treated with cooling or smearing, these are hidden under UV fluctuations and a filter is needed to observe them. Using the low-lying Dirac modes has the advantage over smearing or cooling techniques, that the gauge field is not altered. Also lowlying modes of the covariant Laplace operator have been implemented as a low pass filter [10].

The newly developed formula (9) for the Polyakov loop might be particularly useful for analyzing properties of so-called Kraan-van Baal solutions [1]. There an object of topological charge 1 is made from $n$ constituents (for gauge group $\mathrm{SU}(n)$ ) and the local Polyakov loop is expected to show a specific pattern at the positions of the constituents. For cooled configurations this pattern has been confirmed [12]. The expression (9) can be used to study the local behavior of the Polyakov loop directly for thermalized configurations using low eigenmode filtering techniques. Concerning the dominant contributions, for the local version $L(\vec{x})$ the situation is different from the summed Polyakov loop $P$. In the spectral sum (9) the eigenvalues are multiplied with the densities $\rho(\vec{x})_{\lambda}$ and $\rho(\vec{x})_{\lambda_{ \pm}}$. For the low-lying eigenvectors these densities are known to be large for positions where topological objects are located, while for larger values of $\lambda, \lambda_{ \pm}$the densities are dominated by fluctuations. Numerical tests of using (9) as an infrared filter are in preparation.

\section{Concluding remarks}

How chiral symmetry breaking reflects itself in spectral properties of the Dirac operator is well understood. The chiral condensate is related to the density of eigenvalues near the origin and random matrix theory can be used to describe the behavior of the low-lying eigenvalues.

Also confinement should have a signature in spectral properties of the Dirac operator, which, however, is only vaguely understood so far. In this letter we have shown that the Polyakov loop can be expressed through moments of the Dirac eigenvalues computed with different boundary conditions. At the phase transition of pure gauge theory the Polyakov loop acquires a non-vanishing expectation value and the dependence of these moments on the boundary condition changes. We also discuss correlators of local Polyakov loops which are related to the potential between static quarks. Based on this relation we suggest that in the confining phase correlators of Dirac eigenvector densities decay exponentially with spatial separation, while a power law should be seen in the deconfined phase.

Some of our spectral sums might get sizable contributions from large eigenvalues. Analyzing the ultraviolet part of the Dirac spectrum is a challenge both for numerical and analytical approaches. The results presented here are certainly only an initial step indicating which spectral quantities can be studied. The ambitious goal of such a study could be a link between the spectral properties in the infrared, which are connected to chiral symmetry breaking, and the ultraviolet part probably more relevant for confinement.

\section{Acknowledgments}

The author thanks Falk Bruckmann, Tom DeGrand, Antonio Garcia-Garcia, Christian Lang, Michael MüllerPreussker, Kim Splittorff and Pierre van Baal for valuable discussions and remarks. We thank the Institute for Nuclear Theory at the University of Washington, where part of this work was done, for its hospitality and the Department of Energy for support during that stay.
[1] T. Banks and A. Casher, Nucl. Phys. B169, 103 (1980).

[2] T. Schäfer, E.V. Shuryak, Rev. Mod. Phys. 70, 323 (1998); D. Diakonov, Prog. Part. Nucl. Phys. 51, 173 (2003).

[3] L. McLerran, B. Svetitsky, Phys. Rev. D 24, 450 (1981).

[4] P. Hasenfratz, F. Karsch, Phys. Lett. B 125, 308 (1983).

[5] P.H. Ginsparg, K.G. Wilson, Phys. Rev. D 25, 2649 (1982).

[6] M. Golterman, Y. Shamir, Phys. Rev. D 68, 074501 (2003); M. Golterman, Y. Shamir, B. Svetitsky, Phys. Rev. D 71 (2005) 071502; B. Svetitsky, Y. Shamir, M. Golterman, PoS LAT2005, 129 (2006).

[7] C. Gattringer, S. Schaefer, Nucl. Phys. B 654, 30 (2003); C. Gattringer, P.E.L. Rakow, A. Schäfer, W. Söldner, Phys. Rev. D 66, 054502 (2002).

[8] C. Gattringer, Phys. Rev. D 63, 114501 (2001); C. Gattringer, I. Hip, C.B. Lang, Nucl. Phys. B597, 451 (2001).

[9] I. Horvath et al., Phys. Rev. D 66, 034501 (2002); Phys.
Rev. D 67, 011501 (2003); C. Gattringer, Phys. Rev. Lett. 88 (2002) 221601; C. Aubin et al. [MILC Collaboration], Nucl. Phys. Proc. Suppl. 140, 626 (2005); J. Gattnar et al., Nucl. Phys. B 716, 105 (2005); C. Gattringer et al., Nucl. Phys. B 617, 101 (2001); Nucl. Phys. B 618, 205 (2001); F.V. Gubarev, S.M. Morozov, M.I. Polikarpov, V.I. Zakharov, hep-lat/0505016

[10] J. Greensite et al, Phys. Rev. D71, 114507 (2005); F. Bruckmann, E.-M. Ilgenfritz, Phys. Rev. D 72, 114502 (2005); PoS LAT2005, 305 (2005); Nucl. Phys. Proc. Suppl. 153, 33 (2006).

[11] T.C. Kraan, P. van Baal, Phys. Lett. B 428 (1998) 268; B 435 (1998) 389, Nucl. Phys. B 533 (1998) 627; K. Lee, C. Lu, Phys. Rev. D 58 (1998) 1025011.

[12] E.-M. Ilgenfritz et al, Phys. Rev. D 66 (2002) 074503. 